\title{
CHARACTER RIGIDITY OF SIMPLE ALGEBRAIC GROUPS
}

\author{
BACHIR BEKKA
}

\begin{abstract}
We prove the following extension of Tits' simplicity theorem. Let $k$ be an infinite field, $G$ an algebraic group defined and quasi-simple over $k$, and $G(k)$ the group of $k$-rational points of $G$. Let $G(k)^{+}$be the subgroup of $G(k)$ generated by the unipotent radicals of parabolic subgroups of $G$ defined over $k$ and denote by $P G(k)^{+}$the quotient of $G(k)^{+}$by its center.

Then every normalized function of positive type on $P G(k)^{+}$ which is constant on conjugacy classes is a convex combination of $\mathbf{1}_{P G(k)^{+}}$and $\delta_{e}$. As corollary, we obtain that, when $k$ is countable, the only ergodic IRS's (invariant random subgroups) of $P G(k)^{+}$ are $\delta_{P G(k)^{+}}$and $\delta_{\{e\}}$. A further consequence is that, when $k$ is a global field and $G$ is $k$-isotropic and has trivial center, every measure preserving ergodic action of $G(k)$ on a probability space either factorizes through the abelianization $G(k)_{\mathrm{ab}}$ or is essentially free.
\end{abstract}

\section{INTRODUCTION}

Given a locally compact group $G$, recall that a continuous function $\varphi: G \rightarrow \mathbf{C}$ is of positive type (or positive definite) if, for all $g_{1}, \ldots, g_{n} \in G$, the matrix $\left(\varphi\left(g_{j}^{-1} g_{i}\right)\right)_{1 \leq i, j \leq n}$ is positive semi-definite. As is well-known, such functions are exactly the diagonal matrix coefficients of unitary representations of $G$ in Hilbert spaces (see Section 2). The set $P(G)$ of functions $\varphi$ of positive type on $G$, normalized by the condition $\varphi(e)=1$, is convex and the extreme points in $P(G)$ are the diagonal matrix coefficients, given by unit vectors, of irreducible unitary representations of $G$.

Assume now that $G$ is a discrete group. By results of Glimm Gli61] and Thoma [Tho68], the classification of the unitary dual $\widehat{G}$, that is,

2010 Mathematics Subject Classification: 20G05, 22D10, 22D25, 22D40

Date: May 14, 2020.

The author acknowledges the support by the ANR (French Agence Nationale de la Recherche) through the projects Labex Lebesgue (ANR-11-LABX-0020-01) and GAMME (ANR-14-CE25-0004). 
the set of irreducible unitary representations of $G$ up to unitary equivalence, is hopeless, unless $G$ is virtually abelian. However, the set of characters or traces of $G$, that we are going to define, seems to be more tractable.

Definition 1.1. A function $\varphi \in P(G)$ is a trace on $G$ if $\varphi$ is central (that is, constant on conjugacy classes of $G$ ). The set $\operatorname{Tr}(G)$ of traces on $G$ is a convex compact subset of the unit ball of $\ell^{\infty}(G)$ for the topology of pointwise convergence. An extreme point of $\operatorname{Tr}(G)$ is a character of $G$. We denote by $\operatorname{Char}(G)$ the set of characters of $G$.

Several authors studied the problem of the description of $\operatorname{Char}(G)$ for various discrete groups (see e.g. [Tho64b], [Kir65], Ovč71], [Sku76], [Bek07], DM14], Pet14], [PT16]).

Traces on groups arise in various settings. An immediate example of a trace on $G$ is the usual normalized trace associated to a finite dimensional unitary representation of $G$. More generally, let $(\pi, \mathcal{H})$ be a unitary representation of $G$ such that the von Neumann algebra $\pi(G)^{\prime \prime}$ of $\mathcal{B}(\mathcal{H})$ generated by $\pi(G)$ admits a finite trace $\tau$. Then $\varphi_{\pi}=\tau \circ \pi$ belongs to $\operatorname{Tr}(G)$; moreover, $\varphi_{\pi} \in \operatorname{Char}(G)$ if and only if $\pi(G)^{\prime \prime}$ is a factor. Conversely, every $\varphi \in \operatorname{Tr}(G)$ is of the form $\varphi_{\pi}$ for $\pi$ as above; for all this, see [Dix77, Chap. 17, §. 3].

Another example of a trace on $G$ is the characteristic function $\mathbf{1}_{N}$ of a normal subgroup $N$ of $G$. Indeed, $\mathbf{1}_{H}$ belongs to $P(G)$ for any subgroup $H$ of $G$, since $\mathbf{1}_{H}$ is the matrix coefficient associated to the natural representation of $G$ on $\ell^{2}(G / H)$ and the unit vector $\delta_{H} \in \ell^{2}(G / H)$.

In recent years, there has been a strong interest in the notion of invariant random subgroups, introduced in [AGV14] (see e.g. $\mathrm{ABB}^{+} 17$ ], [Bow15, Gla17], Ver11]). The space $\operatorname{Sub}(G)$ of subgroups of $G$, equipped with the topology induced from $\{0,1\}^{G}$, is a compact space on which $G$ acts by conjugation. An invariant random subgroup (or shortly IRS) of $G$ is a $G$-invariant probability measure on $\operatorname{Sub}(G)$. The Dirac measures on $\operatorname{Sub}(G)$ are given by normal subgroups of $G$, which may be viewed as trivial IRS's. Every IRS $\mu$ defines a trace $\varphi_{\mu}$ on $G$ by

$$
\varphi_{\mu}(g)=\mu(\{H \in \operatorname{Sub}(G) \mid g \in H\}) \quad \text { for all } g \in G ;
$$

indeed, $\varphi_{\mu}$, which is clearly $G$-invariant, belongs to $P(G)$ since $\varphi_{\mu}=$ $\int_{\operatorname{Sub}(G)} \mathbf{1}_{H} d \mu(H)$ is a convex combination of functions of positive type. Examples of IRS's arise as follows: let $G \curvearrowright(X, \nu)$ be a measure preserving action on a probability space $(X, \nu)$; the image $\mu$ of $\nu$ under the map $X \rightarrow \operatorname{Sub}(G), x \mapsto G_{x}$ is an IRS, where $G_{x}$ is the stabilizer of $x$ in $G$. In fact, as shown in AGV14, every IRS is of this form. 
An early important result in this context is the work [SZ94, in which it was shown that, if $G$ is a lattice in a higher rank simple Lie group and $G \curvearrowright(X, \mu)$ an ergodic faithful action with $\mu$ non atomic, then $G \curvearrowright(X, \mu)$ is essentially free (that is, $\varphi_{\mu}=\delta_{e}$ ). For a survey on IRS's, see Gel18].

Let $k$ be a field, $G$ an algebraic group defined and quasi-simple over $k$, and $G(k)$ the group of $k$-rational points of $G$. Let $G(k)^{+}$be the (normal) subgroup of $G(k)$ generated by the unipotent radicals of parabolic subgroups of $G$ defined over $k$. (In case $k$ is perfect, $G(k)^{+}$coincides with the subgroup generated by all unipotent elements in $G(k)$; see [BT73, §.6].) The celebrated Tits simplicity theorem [Tit64] asserts that, when $k$ has at least 4 elements, every subgroup of $G(k)$ which is normalized by $G(k)^{+}$either is contained in the center $Z(G)$ of $G$, or contains $G(k)^{+}$; in particular,

$$
P G(k)^{+}:=G(k)^{+} / Z\left(G(k)^{+}\right)
$$

is a simple group, where $Z\left(G(k)^{+}\right)$is the center of $G(k)^{+}$. We prove the following generalization of this result in the case where $k$ is infinite.

Given a group $H$ and a normal subgroup $N$, we identify $P(H / N)$ with the subset of $P(H)$ of all $\varphi \in P(H)$ with $\left.\varphi\right|_{N}=\mathbf{1}_{N}$; moreover, for a function $\chi: N \rightarrow \mathbf{C}$, we denote by $\widetilde{\chi}$ its trivial extension to $H$, defined by $\tilde{\chi}=\chi$ on $N$ and $\tilde{\chi}=0$ on $H \backslash N$.

Theorem A. Let $k$ be an infinite field and $G$ an algebraic group defined and quasi-simple over $k$. Let $\varphi \in P(G(k))$ be a function of positive type on $G(k)$ which is conjugation-invariant under $G(k)^{+}$. Then $\varphi$ is a convex combination of functions $\varphi_{1}$ and $\varphi_{2}$ in $P(G(k))$ with $\varphi_{1}=1$ on $G(k)^{+}$and $\varphi_{2}=0$ outside $Z(G)(k)$. In particular, we have

$$
\operatorname{Char}(G(k))=\operatorname{Char}\left(G(k) / G(k)^{+}\right) \cup\{\tilde{\chi} \mid \chi \in \widehat{Z(G)(k)}\},
$$

where $\widehat{Z(G)(k)}$ is the dual group of $Z(G)(k)$.

Our proof of Theorem $\mathrm{A}$ is not independent of Tits' simplicity theorem; its overall strategy, similar to the one for the proof of the HoweMoore theorem ([HM79, Theorem 5.1]), proceeds by the analysis of the restriction of a function $\varphi$ as above to the semi-direct product of a maximal $k$-split torus in $G$ with various root spaces. In this analysis, a crucial tool is played by Proposition 2.2 below, which, combined with Tits' simplicity theorem, yields a mixing result of traces on such a semi-direct product.

The following result is an immediate consequence of Theorem $\mathrm{A}$ (see Section 5). 
Corollary B. Let $k$ and $G$ be as in Theorem $A$. Then

$$
\operatorname{Char}\left(G(k)^{+}\right)=\left\{\mathbf{1}_{G(k)^{+}}\right\} \cup\left\{\tilde{\chi} \mid \chi \in Z \widehat{\left(G(k)^{+}\right)}\right\} .
$$

In particular, we have $\operatorname{Char}\left(P G(k)^{+}\right)=\left\{\mathbf{1}_{P G(k)^{+}}, \delta_{e}\right\}$.

Corollary B can easily be extended to the case of semi-simple groups (see Section 51).

Remark 1.2. The result in Corollary $\mathrm{B}$ was obtained in the case $G=$ $S L_{n}$ for $n \geq 3$ in Kir65 and extended in Ovč71] to any Chevalley group $G$ (in this case, $G$ is $k$-split), not of type $A_{1}$ or $B_{2}$ (see however the footnote in op. cit.). The case $G=S L_{2}$ was treated in [PT16].

As a consequence of Corollary $\mathrm{B}$, we obtain the following rigidity result for factor representations of $P G(k)^{+}$and for its IRS's.

Corollary C. Let $k$ and $G$ be as in Theorem $A$.

(i) Let $M$ be a von Neumann factor which possesses a finite trace and $\pi: P G(k)^{+} \rightarrow U(M)$ a homomorphism in the unitary group of $M$ such that $\pi\left(P G(k)^{+}\right)^{\prime \prime}=M$. Then either $\pi$ is the identity homomorphism or $\pi$ extends to an isomorphism $L(G(k)) \rightarrow M$, where $L\left(P G(k)^{+}\right)$is the von Neumann algebra $\lambda\left(P G(k)^{+}\right)^{\prime \prime}$ generated by the regular representation $\lambda$ of $P G(k)^{+}$on $\ell^{2}\left(P G(k)^{+}\right)$.

(ii) Assume that $k$ is countable. The only ergodic IRS's of $P G(k)^{+}$ are $\delta_{e}$ and $\delta_{P G(k)^{+}}$.

Remark 1.3. (i) Item (i) of Corollary $[$ generalizes a classical result of Segal and von Neumann [SvN50] which deals with the case $k=\mathbf{R}$, under a measurability condition on $\pi$ (see also Item (iii) below and Proposition 5.11).

(ii) Assume that $G$ is $k$-isotropic, that is, $G(k)^{+} \neq\{e\}$ (of course, the previous results have content only in this case). Then $G(k)^{+}$is "large", in the sense that $G(k)^{+}$is Zariski dense in $G$ (see [Tit64, 3.2(20)]). In fact, the so-called Whitehead group $W(k, G):=G(k) / G(k)^{+}$is known to be trivial for various groups $G$ and various fields $k$ (for this, see [Tit78], [PR94], Gil09]). Although $W(k, G)$ can be non trivial (even when $G$ is simply connected), it seems that no example is known where $W(k, G)$ is not abelian.

(iii) Let $k$ be either a local field (that is, a non discrete locally compact field) or a global field (that is, either a number field or a function field in one variable over a finite field). It is known that $W(k, G)$ is trivial in both cases, when $G$ is simply 
connected, $k$-isotropic and almost $k$-simple (see PR94, Chap. 7] and [Gil09, Theorem 8.1]). It follows from this and from [BT73, Corollaire 6.5] that $W(k, G)$ is an abelian group, when $G$ is any $k$-isotropic and almost $k$-simple group; since $G(k)^{+}$is perfect ([Tit64, 3.3]), this means that $W(k, G)$ coincides with the abelianization $G(k)_{\text {ab }}$ of $G(k)$. More is known about the structure of $W(k, G)$, when $k$ is a local field (see BT73, 6.14, 6.15]); for instance, $G(\mathbf{R})^{+}$coincides with the identity component of the Lie group $G(\mathbf{R})$.

(iv) Observe that the Zariski-density of $G(k)^{+}$in $G$ implies that the center of $G(k)^{+}$coincides with $Z(G) \cap G(k)^{+}$.

When $k$ is a local field, $G(k)$ has a natural locally compact topology induced by $k$; however, $\operatorname{Char}(G(k))$ in the statement of the following corollary refers to the characters of $G(k)$ as discrete group.

Corollary D. Let $k$ be a local or global field and $G$ a $k$-isotropic and almost $k$-simple algebraic group.

(i) We have $\operatorname{Char}(G(k))=\widehat{G(k)_{\mathrm{ab}}} \cup\{\tilde{\chi} \mid \chi \in \widehat{Z(G)(k)}\}$.

(ii) Assume that $k$ is a global field and that $Z(G)=\{e\}$. Every measure preserving ergodic action of $G(k)$ on a probability space either factorizes through $G(k)_{\mathrm{ab}}$ or is essentially free.

This paper is organized as follows. In Section 2, we prove the mixing result (Proposition 2.2) which is crucial for our approach. Some general facts about relative root spaces of simple algebraic groups are recalled in Section 3. The proofs of Theorem $\mathrm{A}$ and its corollaries are given in Sections 4 and 5 .

Acknowledgement. We are grateful to Jesse Peterson for a useful suggestion concerning the proof of Corollary D,ii.

\section{A PRELIMINARY RESUlt ON TRACES}

Let $G$ be a group. Recall that a unitary representation $(\pi, \mathcal{H})$ of $G$ is a homomorphism $\pi$ from $G$ to the group of unitary operators on a Hilbert space $\mathcal{H}$. The von Neumann algebra $\pi(G)^{\prime \prime}$ generated by $\pi(G)$ is the bicommutant of the subset $\pi(G)$ of the algebra $\mathcal{B}(\mathcal{H})$ of bounded linear operators on $\mathcal{H}$ and can also be described as the closure of the linear span of $\pi(G)$ in the strong (or the weak) operator topology.

Let $\varphi \in P(G)$. Recall (see [BHV08, Appendix C]) that there exists a so-called GNS-triple $(\pi, \mathcal{H}, \xi)$ associated to $\varphi$ which consists of a unitary representation $\pi$ of $G$ on a Hilbert space $\mathcal{H}$ and a unit vector $\xi \in \mathcal{H}$ which is $G$-cyclic (that is, the linear span of $\{\pi(g) \xi \mid g \in G\}$ is dense in $\mathcal{H}$ ) and such that $\varphi(g)=\langle\pi(g) \xi \mid \xi\rangle$ for every $g \in G$. 
The proof of the following lemma is straightforward.

Lemma 2.1. Let $G^{+}$be a subgroup of $G$ and assume that $\varphi$ is $G^{+}$invariant, that is, $\varphi\left(g x g^{-1}\right)=\varphi(x)$ for all $x \in G$ and $g \in G^{+}$. Let $(\pi, \mathcal{H}, \xi)$ be a GNS-triple associated to $\varphi$ and let $\tau_{\varphi}$ be the linear functional on $\pi(G)^{\prime \prime}$ defined by

$$
\tau_{\varphi}(T)=\langle T \xi \mid \xi\rangle \quad \text { for all } \quad T \in \pi(G)^{\prime \prime} .
$$

We have $\tau_{\varphi}(T S)=\tau_{\varphi}(S T)$ for all $T \in \pi(G)^{\prime \prime}$ and $S \in \pi\left(G^{+}\right)^{\prime \prime}$.

If $\theta$ is an automorphism of an abelian locally compact group $U$, we denote by $\chi \mapsto \chi^{\theta}:=\chi \circ \theta^{-1}$ the dual action of $\theta$ on the dual group $\widehat{U}$.

Let $(\pi, \mathcal{H})$ be a unitary representation of a locally compact group $G$. For a subset $X$ of $G$, we denote by $\mathcal{H}^{X}$ the subspace of $X$-invariant vectors in $\mathcal{H}$. Moreover, we will say that $\pi$ is mixing if the matrix coefficient $\langle\pi(\cdot) \xi \mid \eta\rangle$ vanishes at infinity on $G$ (that is, belongs to $\left.C_{0}(G)\right)$ for every $\xi, \eta \in \mathcal{H}$.

The following proposition will be a crucial tool in our proof of Theorem A.

Proposition 2.2. Let $G$ be a discrete group. Let $G^{+}$be a normal subgroup of $G$ containing a subgroup $H$ and an abelian subgroup $U$ normalized by $H$ with the following property: there exists a finite subset $F$ of $H$ such that, for every $h \in H \backslash F$ and every $\chi \in \widehat{U} \backslash\left\{\mathbf{1}_{U}\right\}$, we have $\chi^{h} \neq \chi$.

Let $\varphi: G \rightarrow \mathbf{C}$ be a $G^{+}$-invariant normalized function of positive type on $G$ and let $(\pi, \mathcal{H}, \xi)$ be a GNS-triple associated to $\varphi$.

(i) The restriction of $\left.\pi\right|_{H}$ to the orthogonal complement of $\mathcal{H}^{U}$ is a mixing representation of $H$.

(ii) If $H$ is infinite, then $\mathcal{H}^{H}$ is contained in $\mathcal{H}^{U}$.

(iii) If $\mathcal{H}^{U}=\{0\}$, then $\varphi(h u)=0$ for every $h \in H \backslash F$ and $u \in U$.

Proof. Consider the projection-valued measure $E: \mathcal{B}(\widehat{U}) \rightarrow \operatorname{Proj}(\mathcal{H})$ associated to the restriction $\left.\pi\right|_{U}$ of $\pi$ to $U$, where $\mathcal{B}(\widehat{U})$ is the $\sigma$-algebra of Borel subsets of the compact space $\widehat{U}$ and $\operatorname{Proj}(\mathcal{H})$ the set of orthogonal projections on $\mathcal{H}$. The commutant of $\pi(U)$ coincides with the commutant of $\{E(B) \mid B \in \mathcal{B}(\widehat{U})\}$. In particular, the range of $E(B)$ is $\pi(U)$-invariant and $E(B) \in \pi(U)^{\prime \prime}$ for every $B \in \mathcal{B}(\widehat{U})$. Moreover, the subspace $\mathcal{H}^{U}$ coincides with the range of the projection $E\left(\left\{\mathbf{1}_{U}\right\}\right)$ and we have the covariance relation

$$
\pi(h) E(B) \pi\left(h^{-1}\right)=E\left(B^{h}\right) \quad \text { for all } \quad B \in \mathcal{B}(\widehat{U}), h \in H,
$$


where $B^{h}=\left\{\chi^{h} \mid \chi \in B\right\}$; for all this, see Appendix D as well as the proof of Theorem 1.4.5 in BHV08.

Set

$$
X:=\widehat{U} \backslash\left\{\mathbf{1}_{U}\right\}
$$

and fix $h \in H \backslash F$. Since $\chi^{h} \neq \chi$ for every $\chi \in X$, there exists a family $\left(B_{i}\right)_{i \in I}$ of pairwise disjoint Borel subspaces of $X$ with $X=\bigcup_{i \in I} B_{i}$ such that such that

$$
B_{i} \cap B_{i}^{h}=\emptyset \quad \text { for all } \quad i \in I,
$$

by Lemma 2.3 below. By the general properties of a projection-valued measure, we have

(1) $E\left(B_{i}\right) E\left(B_{j}\right)=0$ for all $i \neq j$,

(2) $\sum_{i \in I} E\left(B_{i}\right)=E(X)$, in the strong operator topology, and

(3) $E\left(B_{i}\right) E\left(B_{i}^{h}\right)=0$ for all $i \in I$.

We claim that, for every $g \in G$, we have

$$
\langle\pi(h u) E(X) \pi(g) \xi \mid E(X) \pi(g) \xi\rangle=0 \quad \text { for all } u \in U .
$$

Indeed, let us first prove the formula for $g=e$. Let $u \in U$. By Lemma 2.1 and the relation (1) above, we have

$$
\begin{aligned}
\left\langle\pi(h u) E\left(B_{i}\right) \xi \mid E\left(B_{j}\right) \xi\right\rangle & =\tau_{\varphi}\left(E\left(B_{j}\right) \pi(h u) E\left(B_{i}\right)\right) \\
& =\tau_{\varphi}\left(E\left(B_{i}\right) E\left(B_{j}\right) \pi(h u)\right)=0,
\end{aligned}
$$

for all $i \neq j$. By successively relations $(2),(*)$, and (3), it follows that

$$
\begin{aligned}
\langle\pi(h u) E(X) \xi \mid E(X) \xi\rangle & =\langle\pi(h u) E(X) \xi \mid E(X) \xi\rangle \\
& =\left\langle\pi(h u)\left(\sum_{i \in I} E\left(B_{i}\right) \xi\right) \mid \sum_{i \in I} E\left(B_{i}\right) \xi\right\rangle \\
& =\sum_{i, j \in I}\left\langle\pi(h u) E\left(B_{i}\right) \xi \mid E\left(B_{j}\right) \xi\right\rangle \\
& =\sum_{i \in I}\left\langle\pi(h u) E\left(B_{i}\right) \xi \mid E\left(B_{i}\right) \xi\right\rangle \\
& =\sum_{i \in I}\left\langle E\left(B_{i}^{h}\right) \pi(h u) \xi \mid E\left(B_{i}\right) \xi\right\rangle \\
& =\sum_{i \in I}\left\langle E\left(B_{i}\right) E\left(B_{i}^{h}\right) \pi(h u) \xi \mid \xi\right\rangle \\
& =0 .
\end{aligned}
$$

Assume now that $g \in G$ is arbitrary and consider the normalized function of positive type $\varphi^{g}$ on $G$ defined by $\varphi^{g}(x)=\varphi\left(g^{-1} x g\right)$ for $x \in G$. It is clear that $(\pi, \mathcal{H}, \pi(g) \xi)$ is a GNS-triple associated to $\varphi$. Moreover, as $G^{+}$is normal in $G$, it is readily checked that $\varphi^{g}$ is $G^{+}$-invariant. 
The linear functional on $\pi(G)^{\prime \prime}$ associated to $\varphi^{g}$ as in Lemma 2.1 is given by $\tau_{\varphi^{g}}(T)=\langle T \pi(g) \xi \mid \pi(g) \xi\rangle$ and satisfies $\tau_{\varphi^{g}}(S T)=\tau_{\varphi^{g}}(T S)$ for $T \in \pi(G)^{\prime \prime}$ and $S \in \pi\left(G^{+}\right)^{\prime \prime}$. Replacing $\varphi$ by $\varphi^{g}$ in the previous argument, we see therefore that

$$
\langle\pi(h u) E(X) \pi(g) \xi \mid E(X) \pi(g) \xi\rangle=0,
$$

for all $u \in U$; so, $(* *)$ holds for every $g \in G$.

The relation $(* *)$ shows that $\left.\pi\right|_{H}$, restricted to the closed linear span $V_{g}$ of

$$
\{\pi(h) E(X) \pi(g) \xi \mid h \in H\},
$$

is a mixing representation of $H$, for every $g \in G$. Observe that $\mathcal{K}:=$ $E(X)(\mathcal{H})$ is the orthogonal complement of $\mathcal{H}^{U}$. Since $\xi$ is a cyclic vector for the $G$-representation $\pi$, the linear span of $\{E(X) \pi(g) \xi \mid g \in G\}$ and hence the linear span of $\bigcup_{g \in G} V_{g}$ is dense in $\mathcal{K}$.

Let $\mathcal{F}$ be the set of all families $\left(\mathcal{H}_{i}\right)_{i \in I}$ of pairwise orthogonal $\pi(H)$ invariant closed subspaces $\mathcal{H}_{i}$ of $\mathcal{K}$ such that $\left.\pi\right|_{H}$, restricted to $\oplus_{i \in I} \mathcal{H}_{i}$ is mixing. We partially order $\mathcal{F}$ by inclusion. Observe that $\mathcal{F}$ is non empty (since $\{0\} \in \mathcal{F}$ ) and that every chain in $\mathcal{F}$ has an upper bound. So, by Zorn's lemma, $\mathcal{F}$ has a maximal element $\left(\mathcal{H}_{i}\right)_{i \in I}$. We claim that $\mathcal{K}=\oplus_{i \in I} \mathcal{H}_{i}$. Indeed, assume, by contradiction, that $\mathcal{K}^{\prime}:=\oplus_{i \in I} \mathcal{H}_{i}$ is a proper subspace of $\mathcal{K}$. Then there exists $g \in G$ such that $V_{g}$ is not contained in $\mathcal{K}^{\prime}$. So, the orthogonal projection $P: V_{g} \rightarrow \mathcal{K}^{\perp}$ is non-zero, where $\mathcal{K}^{\prime \perp}$ is the orthogonal complement of $\mathcal{K}^{\prime}$ in $\mathcal{K}$. Since $P$ is $\pi(H)$ equivariant, there exists non-zero $\pi(H)$-invariant closed subspaces $\mathcal{K}_{1}$ of $V_{g}$ and $\mathcal{K}_{2}$ of $\mathcal{K}^{\prime \perp}$ and a $\pi(H)$-equivariant bijective isometry between $\mathcal{K}_{1}$ and $\mathcal{K}_{2}$ (see BHV08, Proposition A.1.4]). Since $\left.\pi\right|_{H}$ restricted to $V_{g}$ is mixing, the same holds for the restriction of $\left.\pi\right|_{H}$ to $\mathcal{K}_{2}$. So, $\left(\mathcal{H}_{i}\right)_{i \in I} \cup\left\{\mathcal{K}_{2}\right\} \in \mathcal{F}$, and this is a contradiction. This proves Item (i).

Observe that $\mathcal{H}^{U}$ and $\mathcal{K}$ are $H$-invariant, since $H$ normalizes $U$. If $H$ is infinite, then $H$ has no non-zero invariant vector in $\mathcal{K}$ by (i) and hence $\mathcal{H}^{H} \subset \mathcal{H}^{U}$. This proves Item (ii).

If $\mathcal{H}^{U}=\{0\}$, then $E(X)$ is the identity on $\mathcal{H}$ and Item (iii) follows from Equality $(* *)$.

The following lemma was used in the proof of Proposition 2.2

Lemma 2.3. Let $T: X \rightarrow X$ be a homeomorphism of a Hausdorff topological space $X$. Assume that $T$ has no fixed point in $X$. There exists a family $\left(B_{i}\right)_{i \in I}$ of pairwise disjoint Borel subspaces of $X$ with $X=\bigcup_{i \in I} B_{i}$ and such that $B_{i} \cap T\left(B_{i}\right)=\emptyset$ for every $i \in I$. 
Proof. Let $\mathcal{F}$ be the set of all families $\left\{B_{i} \mid i \in I\right\}$ of pairwise disjoint Borel subspaces $B_{i}$ of $X$ such that $B_{i} \cap T\left(B_{i}\right)=\emptyset$ for every $i \in I$. Let $\leq$ be the partial order on $\mathcal{F}$ given by $\left\{B_{i} \mid i \in I\right\} \leq\left\{B_{j}^{\prime} \mid j \in J\right\}$ if, for every $i \in I$, there exists $j \in J$ such that $B_{i}=B_{j}^{\prime}$.

The set $\mathcal{F}$ is non empty; indeed, the family consisting of the single subset $\{\emptyset\}$ belongs to $\mathcal{F}$. Moreover, every chain in $\mathcal{F}$ has obviously an upper bound and so, by Zorn's lemma, $\mathcal{F}$ has a maximal element $\left\{B_{i} \mid i \in I\right\}$.

We claim that $X=\bigcup_{i \in I} B_{i}$. Indeed, assume, by contradiction, that there exists $x \in X \backslash \bigcup_{i \in I} B_{i}$. Since $x \neq T x$, there an open neighbourhood $U$ of $x$ such that $T(x) \notin U$. Consider the Borel subset $B$ of $X$ given by

$$
B=\left(U \cap\left(X \backslash \bigcup_{i \in I} B_{i}\right)\right) \backslash T^{-1}(U) .
$$

It is clear that $T(B) \cap U=\emptyset$ and hence $T(B) \cap B=\emptyset$. So, the family $\{B\} \cup\left\{B_{i} \mid i \in I\right\}$ belongs to $\mathcal{F}$ and $\left\{B_{i} \mid i \in I\right\} \leq\{B\} \cup\left\{B_{i} \mid i \in I\right\}$ Moreover, $\left\{B_{i} \mid i \in I\right\} \neq\{B\} \cup\left\{B_{i} \mid i \in I\right\}$, since $x \in B$ and $x \notin$ $\bigcup_{i \in I} B_{i}$; this is a contradiction.

We will make repeated use of the following elementary lemma. The commutator of two elements $g, h$ in a group is $[g, h]=g h g^{-1} h^{-1}$.

Lemma 2.4. Let $G$ be a group and $\varphi: G \rightarrow \mathbf{C}$ a function of positive type on $G$. Let $g \in G$. Assume that there exist a sequence $\left(g_{n}\right)_{n \geq 1}$ in $G$ such that

- $\varphi\left(\left[g^{-1}, g_{m}\right]^{-1}\left[g^{-1}, g_{n}\right]\right)=0$ for all $m \neq n$ and

- $\varphi\left(g_{n} g g_{n}^{-1}\right)=\varphi(g)$ for all $n \geq 1$.

Then $\varphi(g)=0$.

Proof. Let $(\pi, \mathcal{H}, \xi)$ be a GNS triple for $\varphi$. Then

$$
\left\langle\pi\left(\left[g^{-1}, g_{n}\right]\right) \xi, \pi\left(\left[g^{-1}, g_{m}\right]\right) \xi\right\rangle=\varphi\left(\left[g^{-1}, g_{m}\right]^{-1}\left[g^{-1}, g_{n}\right]\right)=0
$$

for all $m, n$ with $m \neq n$; this shows that $\left(\pi\left(\left[g^{-1}, g_{n}\right]\right) \xi\right)_{n \geq 1}$ is an orthonormal sequence and therefore weakly converges to 0 in $\mathcal{H}$. The claim follows, since, for every $n \geq 1$,

$$
\varphi(g)=\varphi\left(g_{n} g g_{n}^{-1}\right)=\varphi\left(g\left[g^{-1}, g_{n}\right]\right)=\left\langle\pi\left(\left[g^{-1}, g_{n}\right]\right) \xi, \pi\left(g^{-1}\right) \xi\right\rangle .
$$




\section{Some facts about Simple Algebraic Groups}

Let $k$ be any field, and let $G$ be a reductive algebraic group defined over $k$. Let $G(k)$ be the group of $k$-rational points in $G$. We need to recall a few results about the structure of $G$ and $G(k)$, from Bor91] and [BT65].

For a subgroup $H$ of $G$, we will denote by $H(k)$ the subgroup $H \cap G(k)$ of $G(k)$.

Fix a maximal torus $k$-split torus $S$ in $G$ and a maximal torus $T$ in $G$ defined over $k$ and containing $S$. Let $\Phi=\Phi(T, G) \subset X(T)$ be the root system of $G$ with respect to $T$, where $X(T)$ is the group of rational characters of $T$.

Let ${ }_{k} \Phi=\Phi(S, G) \subset X(S)$ be the set of $k$-roots of $G$ with respect to $S$, that is, the set of all non-trivial characters of $S$ which are the restrictions to $S$ of elements of $\Phi$.

As usual, ${ }_{k} \Phi^{+}$(respectively, ${ }_{k} \Phi^{-}$) denotes the set of all positive (respectively, negative) $k$-roots, for a given ordering of $X(S)$.

Let $\alpha, \beta \in{ }_{k} \Phi^{+}$be distinct proportional positive $k$-roots. Then either $\beta=2 \alpha$ or $\alpha=2 \beta$.

For $\alpha \in \Phi$, let $U_{\alpha}$ be the one-parameter root subgroup of $G$ corresponding to $\alpha$; this is the subgroup characterized by the fact that there exists an isomorphism $\theta_{\alpha}: \mathbf{G}_{a} \rightarrow U_{\alpha}$ such that

$$
t \theta_{\alpha}(x) t^{-1}=\theta_{\alpha}\left(t^{\alpha} x\right) \text { for all } t \in T, x \in \mathbf{G}_{a},
$$

where $\mathbf{G}_{a}$ is the additive group of dimension 1 and where we used the exponential notation to denote the action of $X(T)$ on $T$.

Let $\alpha \in{ }_{k} \Phi$. We denote by $U_{(\alpha)}$ be the group generated by all $U_{\beta}$ for $\beta \in \Phi$ such that the restriction of $\beta$ to $S$ is a positive integer multiple of $\alpha$.

Set $\alpha^{\prime}=\alpha$ if $2 \alpha$ is not a $k$-root and $\alpha^{\prime}=2 \alpha$ if $2 \alpha$ is a $k$-root. Then $U_{\left(\alpha^{\prime}\right)}$ and $U_{(\alpha)} / U_{\left(\alpha^{\prime}\right)}$ have a vector space structure over $k$ which coincides with their group structure, such that the action of $t \in S$ on $U_{\left(\alpha^{\prime}\right)}$ and

$U_{(\alpha)} / U_{\left(\alpha^{\prime}\right)}$ is given by scalar multiplication by $t^{\alpha^{\prime}}$ and $t^{\alpha}$, respectively. When $2 \alpha$ is a $k$-root, $U_{(\alpha)}$ is not commutative and its center is $U_{\left(\alpha^{\prime}\right)}$; moreover, $U_{(\alpha)}$ is $k$-isomorphic, as variety, to an affine space.

Let $U^{+}$(respectively $U^{-}$) be the the subgroup generated by all the $U_{(\alpha)}$ for $\alpha \in{ }_{k} \Phi^{+}$(respectively, $\alpha \in{ }_{k} \Phi^{-}$). Let $Z_{G}(S)$ be the centralizer of $S$ in $G$ and $N_{G}(S)$ its normalizer. The subgroups

$$
P^{+}=Z_{G}(S) \ltimes U^{+} \text {and } P^{-}=Z_{G}(S) \ltimes U^{-}
$$


are minimal $k$-parabolic subgroups of $G$ with unipotent radical $U^{+}$ and $U^{-}$, respectively; moreover, we have $P^{+} \cap P^{-}=Z_{G}(S)$. All the subgroups introduced above are defined over $k$.

The Weyl group ${ }_{k} W=N_{G}(S) / Z_{G}(S)$ is finite and every element $w \in_{k} W$ has a representative in $N_{G}(S)(k)$, denoted by the same letter w. So,

$$
{ }_{k} W=N_{G}(S)(k) / Z_{G}(S)(k)
$$

observe that ${ }_{k} W$ acts on $S$, on $X(S)$, and linearly on the real vector space $X(S) \otimes \mathbf{R}$. We equip $X(S) \otimes \mathbf{R}$ with an invariant inner product.

The set ${ }_{k} \Phi$ of $k$-roots is a (possibly non reduced) irreducible root system in $X(S) \otimes \mathbf{R}$, whose Weyl group is isomorphic to ${ }_{k} W$.

We recall the following fundamental result (see Bor91, Theorem $21.15])$.

Theorem 3.1. (Bruhat decomposition) We have

$$
G(k)=\coprod_{w \in \epsilon_{k} W} P^{+}(k) w P^{+}(k)=\coprod_{w \in \epsilon_{k} W} P^{-}(k) w P^{-}(k) .
$$

The following proposition is a special case of BT65, Proposition 3.11].

The set of non-divisible roots is the subset ${ }_{k} \Phi_{\text {nd }}$ of ${ }_{k} \Phi$ of roots $\alpha$ such that $\alpha / 2$ is not a root.

Proposition 3.2. Let $H$ be a connected algebraic subgroup of $U^{+}$normalized by the torus $S$. Then the multiplication map

$$
H \cap U_{\left(\alpha_{1}\right)} \times \cdots \times H \cap U_{\left(\alpha_{n}\right)} \rightarrow H
$$

is an isomorphism of algebraic varieties, where $\alpha_{1}, \ldots, \alpha_{n}$ is any ordering of the set ${ }_{k} \Phi_{\mathrm{nd}}^{+}$. In particular, $U^{+}$is $k$-isomorphic, as variety, to an affine space.

We will need to determine the structure of the center $Z\left(U^{+}\right)$of the unipotent radical $U^{+}$of $P^{+}$.

The set of non-multipliable roots is the subset ${ }_{k} \Phi_{\mathrm{nm}}$ of ${ }_{k} \Phi$ of roots $\alpha$ such that $2 \alpha$ is not a root; so

$$
{ }_{k} \Phi_{\mathrm{nm}}=\left\{\alpha^{\prime} \mid \alpha \in{ }_{k} \Phi\right\},
$$

where $\alpha^{\prime}$ is defined as above for a $k$-root $\alpha$. It is clear that ${ }_{k} \Phi_{\mathrm{nm}}$ is an irreducible and reduced root system in $X(S) \otimes \mathbf{R}$.

Let $R$ be an irreducible and reduced root system in an euclidean space. Recall that $R$ has two lengths of roots when $R$ is of type $B_{n}, C_{n}$ for $n \geq 2$ as well as $F_{4}$ and $G_{2}$ and that $R$ has one length of roots in all other cases (see [Bou68, Chap. VI]). 
Proposition 3.3. Let $\alpha_{l}^{\prime}$ be the highest positive root of the root system ${ }_{k} \Phi_{\mathrm{nm}}$; when ${ }_{k} \Phi_{\mathrm{nm}}$ has two lengths of roots, let $\alpha_{s}^{\prime}$ be its highest short positive root.

- We have $Z\left(U^{+}\right)=U_{\left(\alpha_{l}^{\prime}\right)} U_{\left(\alpha_{s}^{\prime}\right)}$, when the characteristic of $k$ is 2 and ${ }_{k} \Phi$ of type $B_{n}, C_{n}$ or $F_{4}$ as well as when the characteristic of $k$ is 3 and ${ }_{k} \Phi$ of type $G_{2}$.

- We have $Z\left(U^{+}\right)=U_{\left(\alpha_{l}^{\prime}\right)}$ in all other cases.

In particular, the algebraic subgroup $Z\left(U^{+}\right)$is connected and defined over $k$.

Proof. The result is proved in [LP11, Proposition 8.3] in the case where $S=T$ (that is, when $G$ is $k$-split). The proof in this case is based on an analysis of subsystems of rank 2 of the root system $\Phi=\Phi(T, G)$, in combination with the fact that $Z\left(U^{+}\right)$is the direct product, as algebraic variety, of the root spaces it contains.

Denote by $H$ the subgroup generated by $\left\{U_{(\alpha)} \mid \alpha \in{ }_{k} \Phi_{\mathrm{nm}}^{+}\right\}$. Observe that the center $Z(H)$ of $H$, being a characteristic subgroup of $H$, is normalized by $S$. Using Proposition [3.2, the proof of [LP11, Proposition 8.3] extends without any change to the root system ${ }_{k} \Phi_{\mathrm{nm}}$ and shows that the connected component $Z(H)_{0}$ of $Z(H)$ is either $U_{\left(\alpha_{l}^{\prime}\right)}$ or $U_{\left(\alpha_{l}^{\prime}\right)} U_{\left(\alpha_{s}^{\prime}\right)}$, depending on the type of ${ }_{k} \Phi_{\mathrm{nm}}$ and the characteristic of $k$, as described above. Since $U_{\left(\alpha^{\prime}\right)}$ is the center of $U_{(\alpha)}$ for every $\alpha \in{ }_{k} \Phi$, we have $Z(H)=Z\left(U^{+}\right)$.

It remains to show that $Z\left(U^{+}\right)$is connected. Let $U^{*}$ be the product of the $U_{(\alpha)}$, for $\alpha \in{ }_{k} \Phi_{\text {nd }}^{+}$with $\alpha^{\prime} \neq \alpha_{l}^{\prime}$ or $\alpha^{\prime} \notin\left\{\alpha_{l}^{\prime}, \alpha_{s}^{\prime}\right\}$. By Proposition 3.2 again and the result just proved, the multiplication map

$$
Z\left(U^{+}\right)_{0} \times U^{*} \rightarrow U^{+}
$$

is an isomorphism of varieties. Since $Z\left(U^{+}\right)_{0}$ has finite index in $Z\left(U^{+}\right)$, the subgroup $U^{*} \cap Z\left(U^{+}\right)$is finite. As $S$ is connected, it follows that $S$ acts trivially on $U^{*} \cap Z\left(U^{+}\right)$. This implies that $U^{*} \cap Z\left(U^{+}\right)=\{e\}$ and therefore $Z\left(U^{+}\right)=Z\left(U^{+}\right)_{0}$.

Remark 3.4. (i) With the notation as in Proposition 3.3, assume that the root system ${ }_{k} \Phi$ is not reduced. Then ${ }_{k} \Phi$ is of type $B C_{n}$ and so has three lengths of roots. As can be checked, the root system ${ }_{k} \Phi_{\mathrm{nm}}$ is of type $C_{n}$ and its short roots are the roots of ${ }_{k} \Phi$ of intermediate length.

(ii) The same description as in Proposition 3.3 holds for the center $Z\left(U^{-}\right)$of $U^{-}$, with $U_{\left(-\alpha_{l}^{\prime}\right)}$ and $U_{\left(-\alpha_{s}^{\prime}\right)}$ in place of $U_{\left(\alpha_{l}^{\prime}\right)}$ and $U_{\left(\alpha_{s}^{\prime}\right)}$.

We will need to consider reductive subgroups of $G$ attached to a $k$-root. 
Proposition 3.5. Let $\alpha \in{ }_{k} \Phi$ and $G_{(\alpha)}$ the subgroup of $G$ generated by $U_{(\alpha)}, Z_{G}(S)$ and $U_{(-\alpha)}$.

(i) $G_{(\alpha)}$ is a reductive group defined over $k$ and the set $\Phi\left(S, G_{(\alpha)}\right)$ of $k$-roots of $S$ on $G_{(\alpha)}$ is $\mathbf{Z} \alpha \cap_{k} \Phi$.

(ii) Let $\sigma_{\alpha} \in{ }_{k} W$ be the reflection with respect to the hyperplane orthogonal to $\alpha$. Then $\sigma_{\alpha}$ has a representative in $N_{G}(S) \cap G_{(\alpha)}(k)$ and we have

$$
\begin{aligned}
& G_{(\alpha)}(k)=P_{\alpha}^{+}(k) \amalg U_{(\alpha)}(k) \sigma_{\alpha} P_{\alpha}^{+}(k)=P_{\alpha}^{-}(k) \amalg U_{(-\alpha)}(k) \sigma_{\alpha} P_{\alpha}^{-}(k) \\
& \text { where } P_{\alpha}^{ \pm}=Z_{G}(S) U_{( \pm \alpha)} .
\end{aligned}
$$

Proof. Item (i) follows from Theorem 3.13 in [BT65, applied to the closed subset $\left\{\beta \in \Phi(T, G)|\beta|_{S} \in \mathbf{Z} \alpha\right\}$ of $\Phi(T, G)$.

The Weyl group of $\Phi\left(S, G_{(\alpha)}\right)$ is $\left\{e, \sigma_{\alpha}\right\}$. Since $N_{G_{(\alpha)}}(S)$ coincides with $N_{G}(S) \cap G_{(\alpha)}$, Item (ii) follows from the result about the Bruhat decomposition (Theorem 3.1), applied to the reductive group $G_{(\alpha)}$.

Let $G(k)^{+}$be the subgroup of $G(k)$ generated by the unipotent radicals of parabolic subgroups of $G$ defined over $k$. Then $G(k)^{+}$is the subgroup generated by $U^{+}(k)$ and $U^{-}(k)$ ([BT73, Proposition 6.2]).

Let $X_{*}(S)$ denote the set of multiplicative one-parameter subgroups in $S$, that is, the set of morphisms $\mathbf{G L}_{1} \rightarrow S$ of algebraic groups. Elements in $X(S)$ and $X_{*}(S)$ are defined over $k$ and both $X(S)$ and $X_{*}(S)$ are free abelian groups of rank equal to $\operatorname{dim} S$. The Weyl group ${ }_{k} W$ acts in the obvious way on $X_{*}(S)$.

For $\chi \in X(S), \lambda \in X_{*}(S)$, we have $\chi \circ \lambda \in X\left(\mathbf{G L}_{1}\right)$ and so $\chi \circ \lambda$ is of the form $x \mapsto x^{r}$ for some $r \in \mathbf{Z}$. The map $X(S) \times X_{*}(S) \rightarrow \mathbf{Z}$ defined by

$$
\langle\chi, \lambda\rangle=r \text { if } \chi \circ \lambda(x)=x^{r} .
$$

is a duality pairing of $\mathbf{Z}$-modules (see [Bor91, Chap.III, 8.6]).

Let $\lambda \in X_{*}(S), \alpha \in{ }_{k} \Phi$, and $r=\langle\alpha, \lambda\rangle$. Set $r^{\prime}=r$ if $\alpha$ is nonmultipliable and $r^{\prime}=2 r$ otherwise. For $x \in k^{*}$, the action of $\lambda(x)$ on $U_{\left(\alpha^{\prime}\right)}$ and $U_{(\alpha)} / U_{\left(\alpha^{\prime}\right)}$, endowed with the vector space structure mentioned above, is given by $\lambda(x) \cdot v=x^{r^{\prime}} v$ for $v \in U_{\left(\alpha^{\prime}\right)}$ and by $\lambda(x) \cdot v=$ $x^{r} v$ for $v \in U_{(\alpha)} / U_{\left(\alpha^{\prime}\right)}$.

\section{Proof of Theorem $\mathrm{A}$}

Let $G(k)$ be the group of $k$-points of an algebraic group $G$ defined and quasi-simple over a field $k$. We assume that $k$ is infinite and, to avoid trivialities, that $G(k)^{+} \neq\{e\}$, that is, the maximal $k$-split torus $S$ has dimension $>0$. 
4.1. Proof of the first statement. Let $\varphi: G(k) \rightarrow \mathbf{C}$ be a normalized function of positive type. Assume that $\varphi$ is $G(k)^{+}$-invariant and let $(\pi, \mathcal{H}, \xi)$ be a GNS-triple associated to $\varphi$.

Since $G(k)^{+}$is normal in $G(k)$, the closed subspaces

$$
\mathcal{K}_{1}:=\mathcal{H}^{G(k)^{+}} \quad \text { and } \quad \mathcal{K}_{2}:=\mathcal{K}_{1}^{\perp}
$$

are $\pi(G)$-invariant. For $i=1,2$, let $\pi_{i}$ be the corresponding subrepresentations of $\pi$ defined on $\mathcal{K}_{i}$. (Observe that $\mathcal{K}_{i}$ may be $\{0\}$.) Writing $\xi=\xi_{1}+\xi_{2}$ with $\xi_{i} \in \mathcal{K}_{i}$, we have therefore

$$
\varphi=\varphi_{1}+\varphi_{2}
$$

where $\varphi_{i}$ is the (not necessarily normalized) function of positive type on $G$ given by $\varphi_{i}(g)=\left\langle\pi(g) \xi_{i} \mid \xi_{i}\right\rangle$. It is clear that $\varphi_{1}$ and hence $\varphi_{2}$ are $G(k)^{+}$-invariant. Obviously, we have $\left.\varphi_{1}\right|_{G(k)^{+}}=\mathbf{1}_{G(k)^{+}}$. In case $\mathcal{K}_{i} \neq\{0\}$, we have $\xi_{i} \neq 0$ and the triple $\left(\pi_{i}, \mathcal{K}_{i}, t_{i} \varphi_{i}\right)$ is a GNS-triple for the normalized function of positive type $t_{i} \varphi_{i}$, where $t_{i}=\left\|\xi_{i}\right\|^{-2}$.

As a result of the previous discussion, upon replacing $\varphi$ by $\varphi_{2}$, we may and will assume in the sequel that $\mathcal{H}^{G(k)^{+}}=\{0\}$. We have then to show that $\varphi=0$ outside $Z(G)(k)$.

For $\lambda \in X_{*}(S)$, we set

$$
H_{\lambda}:=\lambda\left(\mathbf{G L}_{1}\right) \cap G(k)^{+} .
$$

Assume that $\lambda$ is non trivial. Then $\lambda\left(\mathbf{G L}_{1}\right)$ is a one-dimensional subtorus of $S$; since $k$ is infinite, the subgroup $H_{\lambda}$ of $G(k)^{+}$is Zariskidense in $\lambda\left(\mathbf{G L}_{1}\right)$ (see [BT73, Corollaire 6.8]); in particular, $H_{\lambda}$ is infinite. Let $\alpha \in{ }_{k} \Phi$ and $r:=\langle\alpha, \lambda\rangle \in \mathbf{Z}$. Observe that if $r \neq 0$, then

$$
\lambda\left(k^{*}\right) \cap \operatorname{ker} \alpha=\left\{\lambda(x) \mid x \in k^{*} \text { with } x^{r}=e\right\}
$$

and hence $H_{\lambda} \cap \operatorname{ker} \alpha$ is a finite subset of $H_{\lambda}$.

- First step. Let $\alpha \in{ }_{k} \Phi_{n m}$ be a non-multipliable root. Then there exists a one-parameter subgroup $\lambda \in X_{*}(S)$ such that

$$
\mathcal{H}^{U_{(\alpha)}(k)}=H^{H_{\lambda}} \quad \text { and } \quad \mathcal{H}^{U_{(-\alpha)}(k)}=H^{H_{\lambda}} .
$$

Indeed, by [Tit64, 3.1(13)] (see also the beginning of the proof of Théorème 7.2 in [BT65]), there exists a homomorphism $\rho_{\alpha}: S L_{2} \rightarrow G$ defined over $k$, with kernel contained in the center of $S L_{2}$, such that

$$
\rho_{\alpha}\left(\begin{array}{ll}
1 & t \\
0 & 1
\end{array}\right) \in U_{(\alpha)}, \quad \rho_{\alpha}\left(\begin{array}{ll}
1 & 0 \\
s & 1
\end{array}\right) \in U_{(-\alpha)}, \text { and } \rho_{\alpha}\left(\begin{array}{cc}
x & 0 \\
0 & x^{-1}
\end{array}\right) \in S .
$$

Let $L:=\rho_{\alpha}\left(S L_{2}(k)\right)$ and observe that $\varphi \circ\left(\left.\rho_{\alpha}\right|_{S L_{2}(k)}\right)$ is a central function of positive type on $S L_{2}(k)$. It follows from the classification of the 
characters of $S L_{2}(k)$ in [PT16, Theorem 2.4] that

$$
\left.\varphi\right|_{L}=t \mathbf{1}_{L}+(1-t) \varphi^{\prime}
$$

for some $t \in[0,1]$, where $\varphi^{\prime}$ is a function of positive type on $L$ with $\varphi^{\prime}=0$ outside the (finite) center of $L$. Since a similar statement holds for the conjugate function $\varphi^{g}$ for every $g \in G(k)$, this implies (compare with the proof of Proposition 2.2) that the restriction of $\left.\pi\right|_{L}$ to the orthogonal complement of $\mathcal{H}^{L}$ is a mixing representation of $L$. Let $\lambda \in X_{*}(S)$ be defined by $\lambda(x)=\rho_{\alpha}\left(\begin{array}{cc}x & 0 \\ 0 & x^{-1}\end{array}\right)$ and let

$$
V:=\rho_{\alpha}\left(\left\{\left(\begin{array}{ll}
1 & t \\
0 & 1
\end{array}\right) \mid t \in k\right\}\right) .
$$

Since the subgroups $H_{\lambda}$ and $V$ of $L$ are infinite and since $\left.\pi\right|_{L}$ is mixing on $\left(\mathcal{H}^{L}\right)^{\perp}$, we have

$$
\mathcal{H}^{L}=\mathcal{H}^{H_{\lambda}}=\mathcal{H}^{V} .
$$

Observe that $\langle\alpha, \lambda\rangle=2 \neq 0$ and so $F:=H_{\lambda} \cap \operatorname{ker} \alpha$ is finite. Recall that the subgroup $U_{(\alpha)}(k)$ has the structure of a $k$-vector space. For $\chi \in \widehat{U_{(\alpha)}(k)}$ and $x \in k^{*}$, we have

$$
\left.\chi^{\lambda(x)}(u)=\chi\left(\lambda(x)^{-1} \cdot u\right)\right)=\chi\left(x^{-2} u\right) \quad \text { for all } u \in U_{(\alpha)}(k) .
$$

Thus, for every $t \in H_{\lambda} \backslash F$ and every $\chi \in \widehat{U_{(\alpha)}(k)}$ with $\chi \neq \mathbf{1}_{U_{(\alpha)}(k)}$, we have $\chi^{t} \neq \chi$. Proposition 2.2, applied to the subgroup $H_{\lambda} U_{(\alpha)}(k)$ of $G(k)^{+}$, shows then that $\mathcal{H}^{H_{\lambda}}$ is contained in $\mathcal{H}^{U_{(\alpha)}(k)}$.

It is obvious that $\mathcal{H}^{U_{(\alpha)}(k)}$ is contained in $\mathcal{H}^{V}$ and it was shown before that $\mathcal{H}^{V}=\mathcal{H}^{H_{\lambda}}$. As a result, we obtain that $\mathcal{H}^{U_{(\alpha)}(k)}=\mathcal{H}^{H_{\lambda}}$.

The same proof shows also that $\mathcal{H}^{U_{(-\alpha)}(k)}=\mathcal{H}^{H_{\lambda}}$.

- Second step. Let $\alpha \in{ }_{k} \Phi$ be such that $U_{(\alpha)}$ is either contained in the center $Z\left(U^{+}\right)$of $U^{+}$or in the center $Z\left(U^{-}\right)$of $U^{-}$. We claim that $\mathcal{H}^{U_{(\alpha)}(k)}$ is $G(k)$-invariant.

Indeed, assume that $U_{(\alpha)} \subset Z\left(U^{+}\right)$, that is, $\alpha \in{ }_{k} \Phi^{+}$. Observe that $\alpha$ is non-multipliable (see Proposition 3.3). By the first step, there exists a one-parameter subgroup $\lambda \in X_{*}(S)$ such that $\mathcal{H}^{U_{(\alpha)}(k)}=\mathcal{H}^{U_{(-\alpha)}}{ }^{(k)}=$ $H^{H_{\lambda}}$.

Observe that $\mathcal{H}^{H_{\lambda}}$ is obviously invariant under $Z_{G}(S)(k)$. Since $G(k)$ is generated by $U^{+}(k), U^{-}(k)$ and $Z_{G}(S)(k)$, it suffices therefore to show that $\mathcal{H}^{U_{(\alpha)}(k)}$ is invariant under $U_{\left(\alpha^{\prime}\right)}(k)$ for every $k$-root $\alpha^{\prime}$.

Let $\alpha^{\prime} \in{ }_{k} \Phi^{+}$. Since $U_{( \pm \alpha)} \subset Z\left(U^{ \pm}\right)$, it is obvious that $\mathcal{H}^{U_{(\alpha)}(k)}$ is invariant under $U_{\left(\alpha^{\prime}\right)}(k)$ and that $\mathcal{H}^{U_{(-\alpha)}(k)}$ is invariant under $U_{\left(-\alpha^{\prime}\right)}(k)$. 
Since $H_{(\alpha)(k)}^{U_{(\alpha)}}=H_{(-\alpha)}^{U_{(k)}}$, the claim follows in the case where $U_{(\alpha)}$ is contained in $Z\left(U^{+}\right)$.

The case where $U_{(\alpha)}$ is contained in $Z\left(U^{-}\right)$is entirely similar.

- Third step. Let $\alpha \in{ }_{k} \Phi$ be such that $U_{(\alpha)}$ is either contained in $Z\left(U^{+}\right)$or in $Z\left(U^{-}\right)$. We claim that $\mathcal{H}^{U_{(\alpha)}(k)}=\{0\}$.

Indeed, assume, by contradiction, that $\mathcal{H}^{U_{(\alpha)}(k)} \neq\{0\}$. By the second step, $\mathcal{H}^{U_{(\alpha)}(k)}$ is $G(k)$-invariant and so defines a subrepresentation $\rho$ of $\pi$. The kernel $L$ of $\rho$ is a normal subgroup of $G(k)$ containing the non-central subgroup $U_{(\alpha)}(k)$. It follows from Tits' simplicity theorem ([Tit64]) that $L$ contains $G(k)^{+}$. This is a contradiction, since $\mathcal{H}^{G(k)^{+}}=$ $\{0\}$, by our assumption.

In the sequel, we will prove that $\varphi(g)=0$ for elements $g$ from various subsets of $G(k)$; for this, we will repeatedly use Lemma 2.4 by finding sequences $\left(g_{n}\right)_{n}$ in $G(k)^{+}$such that $\varphi\left(\left[g^{-1}, g_{m}\right]^{-1}\left[g^{-1}, g_{n}\right]\right)=0$ for all $m \neq n$.

- Fourth step. Let $\alpha \in{ }_{k} \Phi$ be such that $U_{(\alpha)}$ is either contained in $Z\left(U^{+}\right)$or in $Z\left(U^{-}\right)$. We claim that $\varphi(g)=0$ for every $g \in U_{(\alpha)}(k)$ with $g \neq e$.

Indeed, consider the subgroup $G_{(\alpha)}$ generated by $U_{(\alpha)}, Z_{G}(S)$ and $U_{(-\alpha)}$. We have

$$
G_{(\alpha)}(k)=P_{\alpha}^{-}(k) \amalg U_{(-\alpha)}(k) \sigma_{\alpha} P_{\alpha}^{-}(k),
$$

where $P_{\alpha}^{-}=Z_{G}(S) U_{(-\alpha)}$ and $\sigma_{\alpha}$ is the reflection which sends $\alpha$ to $-\alpha$ (see Proposition 3.5).

Assume that $U_{(\alpha)} \subset Z\left(U^{+}\right)$and let $g \in U_{(\alpha)}(k) \backslash\{e\}$. Since $P_{\alpha}^{+} \cap P_{\alpha}^{-}=$ $Z_{G}(S)$, we have $g \notin P_{\alpha}^{-}$and hence

$$
g \in U_{(-\alpha)}(k) \sigma_{\alpha} P_{\alpha}^{-}(k) .
$$

So, the conjugate of $g$ by some element from $U_{(-\alpha)}(k) \subset G(k)^{+}$belongs to $\sigma_{\alpha} P_{\alpha}^{-}(k)$. We may therefore assume that $g$ is of the form

$$
g=\sigma_{\alpha} \gamma u \text { for some } \gamma \in Z_{G}(S)(k) \text { and } u \in U_{(-\alpha)}(k) .
$$

Choose $\lambda \in X_{*}(S)$ such that $r:=\langle\alpha, \lambda\rangle \neq 0$. The corresponding subgroup $H_{\lambda}$ of $G(k)^{+}$is infinite and we can therefore find a sequence $\left(x_{n}\right)_{n \geq 1}$ in $k^{*}$ such that $\lambda\left(x_{n}\right) \in G(k)^{+}$and such that

$$
x_{m}^{2 r} \neq x_{n}^{2 r} \quad \text { for all } \quad m \neq n .
$$

Consider the multiplicative one-parameter subgroup

$$
\lambda^{\prime}:=\lambda^{\sigma_{\alpha}} \lambda^{-1} \in X_{*}(S)
$$


Observe that $\left\langle\sigma_{\alpha}(\alpha), \lambda\right\rangle=-r$ and hence

$$
\left\langle\alpha, \lambda^{\prime}\right\rangle=\left\langle\sigma_{\alpha}(\alpha), \lambda\right\rangle-\langle\alpha, \lambda\rangle=-2 r
$$

Therefore, we have

$$
\lambda^{\prime}\left(x_{m}\right)^{-1} \lambda^{\prime}\left(x_{n}\right) \notin \operatorname{ker}(-\alpha) \text { for all } m \neq n .
$$

Moreover,

$$
\lambda^{\prime}\left(x_{n}\right)=\lambda^{\sigma_{\alpha}}\left(x_{n}\right) \lambda^{-1}\left(x_{n}\right) \in H_{\lambda^{\prime}} \text { for all } n \geq 1,
$$

since $G(k)^{+}$is a normal subgroup of $G(k)$. By the third step, there is no non-zero $U_{(-\alpha)}(k)$-invariant vector in $\mathcal{H}$. Therefore, Proposition 2.2 applied to $H_{\lambda^{\prime}} U_{(-\alpha)}(k)$ shows that

$(* *) \quad \varphi(h u)=0$ for all $h \in H_{\lambda^{\prime}} \backslash \operatorname{ker}(-\alpha)$ and $u \in U_{(-\alpha)}(k)$.

For every $n \geq 1$, we have

$$
\left[\sigma_{\alpha}^{-1}, \lambda\left(x_{n}\right)\right]=\lambda\left(x_{n}\right)^{\sigma_{\alpha}} \lambda\left(x_{n}\right)^{-1}=\lambda^{\prime}\left(x_{n}\right) .
$$

Set $g_{n}:=\left[g^{-1}, \lambda\left(x_{n}\right)\right]$; then

$$
g_{n}=u^{-1} \gamma^{-1}\left[\sigma_{\alpha}^{-1}, \lambda\left(x_{n}\right)\right] \gamma\left(\lambda\left(x_{n}\right) \cdot u\right)=u^{-1} \lambda^{\prime}\left(x_{n}\right) x_{n}^{-r} u .
$$

In view of $(*)$ and $(* *)$, we have therefore

$$
\varphi\left(g_{m}^{-1} g_{n}\right)=\varphi\left(\lambda^{\prime}\left(x_{m}\right)^{-1} \lambda^{\prime}\left(x_{n}\right)\left(\left(x_{n}^{-r}-x_{m}^{-r}\right) u\right)\right)=0
$$

for all $n \neq m$ and the claim follows.

The case where $U_{(\alpha)} \subset Z\left(U^{-}\right)$is treated similarly.

- Fifth step. We claim that $\varphi(g)=0$ for every $g \in Z\left(U^{ \pm}\right)(k)$ with $g \neq e$.

We will only treat the case of $Z\left(U^{+}\right)$, the case of $Z\left(U^{-}\right)$being entirely similar.

By Proposition 3.3, we have either $Z\left(U^{+}\right)(k)=U_{(\alpha)}^{+}(k)$ or $Z\left(U^{+}\right)(k)=$ $U_{(\alpha)}^{+}(k) U_{(\beta)}^{+}(k)$ for distinct roots $\alpha, \beta \in{ }_{k} \Phi_{\mathrm{nm}}^{+}$. In the first case, the claim follows from the fourth step.

So, we may assume that $Z\left(U^{+}\right)(k)=U_{(\alpha)}(k) U_{(\beta)}(k)$ for roots $\alpha \neq \beta$ as above. Let $g \in Z\left(U^{+}\right)(k)$ with $g \neq e$. Then $g=u v$ for $u \in U_{(\alpha)}(k)$ and $v \in U_{(\beta)}(k)$

If $u=e$, then $g \in U_{(\beta)}(k)$ and therefore $\varphi(g)=0$, by the fourth step. We can therefore assume that $u \neq e$.

Since $\alpha$ and $\beta$ are non proportional, we can find a one-parameter subgroup $\lambda \in X_{*}(S)$ such that

$$
\langle\beta, \lambda\rangle=0 \text { and }\langle\alpha, \lambda\rangle=r \neq 0 .
$$


Choose a sequence $\left(x_{n}\right)_{n \geq 1}$ in $k^{*}$ such that $\lambda\left(x_{n}\right) \in G(k)^{+}$and such that

$$
x_{m}^{r} \neq x_{n}^{r} \quad \text { for all } \quad m \neq n .
$$

We have

$$
\lambda\left(x_{n}\right) \cdot v=v \text { and } \lambda\left(x_{n}\right) \cdot u=x_{n}^{r} u .
$$

and, with $g_{n}:=\left[g^{-1}, \lambda\left(x_{n}\right)\right]$,

$$
g_{n}=v^{-1} u^{-1}\left(x_{n}^{r} u\right) v \quad \text { for all } n \geq 1 .
$$

Therefore, we have, by the fourth step,

$$
\varphi\left(g_{m}^{-1} g_{n}\right)=\varphi\left(v^{-1}\left(x_{m}^{r} u\right)^{-1}\left(x_{n}^{r} u\right) v\right)=\varphi\left(\left(x_{n}^{r}-x_{m}^{r}\right) u\right)=0
$$

for all $m \neq n$ and the claim follows.

For subgroups $A$ and $B$ of a group, $[A, B]$ denotes the subgroup generated by the commutators $[a, b]$ for $a \in A, b \in B$.

Recall that the descending central series $\left(\mathcal{C}^{i}\left(U^{+}\right)\right)_{0 \leq i \leq n}$ of the nilpotent group $U^{+}$is inductively defined by

$$
\mathcal{C}^{0}\left(U^{+}\right)=U^{+} \text {and } \mathcal{C}^{i+1}\left(U^{+}\right)=\left[U^{+}, \mathcal{C}^{i}\left(U^{+}\right)\right] \text {for } 0 \leq i \leq n-1,
$$

where $n$ is the smallest integer $i \geq 1$ with $\mathcal{C}^{i}\left(U^{+}\right)=\{e\}$. Every $\mathcal{C}^{i}\left(U^{+}\right)$ is an algebraic normal subgroup of $U^{+}$defined over $k$.

- Sixth step. We claim that $\varphi(g)=0$ for every $g \in U^{ \pm}(k)$ with $g \neq e$. We will only treat the case of $U^{+}$. Let $i \in\{1, \ldots, n-1\}$. Assume that

$$
\varphi(u)=0 \quad \text { for all } u \in \mathcal{C}^{i+1}\left(U^{+}\right)(k) \backslash\{e\} .
$$

Let $g \in \mathcal{C}^{i}\left(U^{+}\right)(k)$. We are going to show that $\varphi(g)=0$. Once proved, the claim will follow since $\mathcal{C}^{0}\left(U^{+}\right)=U^{+}$and $\mathcal{C}^{n}\left(U^{+}\right)=\{e\}$.

In view of the fifth step, we can assume that $g \notin Z\left(U^{+}\right)$. The map

$$
f: U^{+} \rightarrow U^{+}, \quad u \mapsto\left[g^{-1}, u\right]
$$

is morphism of algebraic varieties and is defined over $k$. Since $g \in$ $\mathcal{C}^{i}\left(U^{+}\right)$and $g \notin Z\left(U^{+}\right)$, the image $f\left(U^{+}\right)$of $f$ is contained in $\mathcal{C}^{i+1}\left(U^{+}\right)$ and is distinct from $\{e\}$. The Zariski-closure of $f\left(U^{+}\right)$is an irreducible $k$-subvariety of $U^{+}$(see proof of [Bor91, 2.2 Proposition]). In particular, the set $f\left(U^{+}\right)$is infinite.

Since $U^{+}(k)$ is Zariski dense in $U^{+}$(see Proposition 3.2 or Theorem 21.20 (i) in [Bor91]), we can find a sequence $\left(u_{n}\right)_{n \geq 1}$ in $U^{+}(k)$ such that $f\left(u_{m}\right) \neq f\left(u_{n}\right)$, that is, such that

$$
\left[g^{-1}, u_{m}\right] \neq\left[g^{-1}, u_{n}\right] \text { for all } n \neq m .
$$

Then

$$
\left[g^{-1}, u_{m}\right]^{-1}\left[g^{-1}, u_{n}\right] \in \mathcal{C}^{i+1}\left(U^{+}\right)(k) \backslash\{e\} ;
$$


hence, by our assumption, we have

$$
\varphi\left(\left[g^{-1}, u_{m}\right]^{-1}\left[g^{-1}, u_{n}\right]\right)=0 \text { for all } m \neq n
$$

and the claim follows.

- Seventh step. Let $t \in S(k)$, with $t \notin Z(G)$. We claim that $\varphi(t)=0$. Since $t \notin Z(G)$, there exists $\alpha \in{ }_{k} \Phi$ such that $t^{\alpha} \neq 1$; indeed, otherwise $t$ would centralize $U^{+}, U^{-}$and $Z_{G}(S)$ and would therefore belong to $Z(G)(k)$.

Since $k$ and hence $U_{(\alpha)}(k)$ is infinite, we can find a sequence $\left(u_{n}\right)_{n \geq 1}$ in $U_{(\alpha)}(k)$ with $u_{n} \neq u_{m}$ for $n \neq m$. We are going to show that

$$
\left[t^{-1}, u_{m}\right] \neq\left[t^{-1}, u_{n}\right] \text { for all } m \neq n \text {. }
$$

Assume first that $\alpha$ is non-multipliable. Then

$$
\left[t^{-1}, u_{m}\right]=\left(t^{-\alpha}-1\right) u_{m} \neq\left(t^{-\alpha}-1\right) u_{n}=\left[t^{-1}, u_{n}\right] \text { for all } m \neq n \text {. }
$$

Assume next that $2 \alpha$ is a root. We see in a similar way that the images of $\left[t^{-1}, u_{m}\right]$ and $\left[t^{-1}, u_{n}\right]$ in $U_{(\alpha)} / U_{(2 \alpha)}$ are distinct.

Therefore, by the fourth step, we have

$$
\varphi\left(\left[t^{-1}, u_{m}\right]^{-1}\left[t^{-1}, u_{n}\right]\right)=0 \text { for all } m \neq n
$$

and the claim follows.

- Eighth step. Let $g \in S(k) U^{ \pm}(k)$ with $g \notin Z(G)$. We claim that $\varphi(g)=0$.

We have $g=t u$ for some $t \in S(k)$ and $u \in U^{ \pm}(k)$. By the sixth and the seventh step, we can assume that $t \neq e$ and that $u \neq e$. We treat only the case where $u \in U^{+}(k)$.

Write $u=\prod_{\alpha \in_{k} \Phi^{+}} u_{\alpha}$, with $u_{\alpha} \in U_{(\alpha)}$ (see Proposition 3.2). Let $\alpha_{0} \in{ }_{k} \Phi^{+}$be such that $u_{\alpha_{0}} \neq e$. Choose $\lambda \in X_{*}(S)$ such that $r:=$ $\left\langle\alpha_{0}, \lambda\right\rangle \neq 0$ and a sequence $\left(x_{n}\right)_{n \geq 1}$ in $k^{*}$ such that $\lambda\left(x_{n}\right) \in G(k)^{+}$and $x_{m}^{r} \neq x_{n}^{r}$ for all $m \neq n$. One checks, as in the seventh step, that

$$
\lambda\left(x_{m}\right) \cdot u_{\alpha_{0}} \neq \lambda\left(x_{n}\right) \cdot u_{\alpha_{0}} \text { for all } n \neq m .
$$

Then

$$
\left[g^{-1}, \lambda\left(x_{m}\right)\right]=u^{-1} \prod_{\alpha \in_{k} \Phi^{+}} \lambda\left(x_{m}\right) \cdot u_{\alpha} \neq u^{-1} \prod_{\alpha \in_{k} \Phi^{+}} \lambda\left(x_{n}\right) \cdot u_{\alpha}=\left[g^{-1}, \lambda\left(x_{n}\right)\right]
$$

hence, by the sixth step,

$$
\varphi\left(\left[g^{-1}, \lambda\left(x_{m}\right)\right]^{-1}\left[g^{-1}, \lambda\left(x_{n}\right)\right]\right)=0 \quad \text { for all } m \neq n
$$

and the claim follows.

- Ninth step. Let $g \in G(k) \backslash Z_{G}(S)$. We claim that $\varphi(g)=0$. 
We follow the strategy used in the fourth step. Recall that $P^{ \pm}=$ $Z_{G}(S) U^{ \pm}$. Since $Z_{G}(S)=P^{+} \cap P^{-}$, we have either $g \notin P^{+}(k)$ or $g \notin P^{-}(k)$. Assume that, say, $g \notin P^{+}(k)$. It follows from the Bruhat decomposition (Theorem 3.1) that $g \in U^{+}(k) w P^{+}(k)$ for some $w \in{ }_{k} W$ with $w \neq e$. So, the conjugate of $g$ by some element from $U^{+}(k) \subset$ $G(k)^{+}$belongs to $w P^{+}(k)$. We can therefore assume that $g$ is of the form

$$
g=w \gamma u \text { for some } \gamma \in Z_{G}(S)(k) \text { and } u \in U^{+}(k) .
$$

Since $w$ is non trivial, there exists $\lambda \in X_{*}(S)$ such that $\lambda^{w^{-1}} \neq \lambda$. So, $\lambda^{\prime}:=\lambda^{w^{-1}} \lambda^{-1}$ is a non trivial one-parameter subgroup in $S$ and we can therefore find a sequence $\left(x_{n}\right)_{n \geq 1}$ in $k^{*}$ such that $\lambda\left(x_{n}\right) \in G(k)^{+}$ and such that

$$
\lambda^{\prime}\left(x_{m}\right)^{-1} \lambda^{\prime}\left(x_{n}\right) \notin Z(G)(k) \text { for all } m \neq n .
$$

We have

$$
\left[w^{-1}, \lambda\left(x_{n}\right)\right]=\lambda^{\prime}\left(x_{n}\right) \text { for all } n \geq 1,
$$

and, setting $u_{n}:=\lambda\left(x_{n}\right) \cdot u \in U^{+}(k)$,

$$
\left[g^{-1}, \lambda\left(x_{n}\right)\right]=u^{-1} \lambda^{\prime}\left(x_{n}\right) u_{n} \text { for all } n \geq 1 .
$$

By the eighth step, it follows that

$$
\varphi\left(\left[g^{-1}, \lambda\left(x_{m}\right)\right]^{-1}\left[g^{-1}, \lambda\left(x_{n}\right)\right]\right)=\varphi\left(\lambda^{\prime}\left(x_{m}\right)^{-1} \lambda^{\prime}\left(x_{n}\right)\left(u_{n} u_{m}^{-1}\right)\right)=0
$$

for all $m \neq n$ and the claim follows.

- Tenth step. Let $g \in Z_{G}(S)$ with $g \notin Z(G)(k)$. We claim that $\varphi(g)=0$.

Indeed, in view of the ninth step, it suffices to show that $G(k) \backslash Z_{G}(S)$ contains a conjugate of $g$ under $G(k)^{+}$. Assume, by contradiction, that every $G(k)^{+}$-conjugate of $g$ is contained in $Z_{G}(S)$. Then the subgroup $L$ generated by the $G(k)^{+}$-conjugates of $g$ is contained in $Z_{G}(S)$. Since $g \notin Z(G)(k)$, Tits' simplicity theorem implies that $L$ contains $G(k)^{+}$. Hence, $G(k)^{+}$is contained in $Z_{G}(S)$. This is a contradiction, since $S$ is non trivial.

4.2. Proof of the second statement. The first statement of Theorem A implies that every $\varphi \in \operatorname{Char}(G(k))$ either factorizes through $G(k) / G(k)^{+}$or is of the form $\tilde{\chi}$ for some $\chi \in \widehat{Z(G)(k)}$.

Conversely, let $\chi \in \widehat{Z(G)(k)}$. We claim that $\tilde{\chi}$, which clearly is a $G(k)$-invariant function from $P(G(k))$, is indecomposable. Let $\varphi_{1}, \varphi_{2}$ be two $G(k)$-invariant functions from $P(G(k))$ and $t \in(0,1)$ such that $\widetilde{\chi}=t \varphi_{1}+(1-t) \varphi_{2}$. 
On the one hand, by the first statement of Theorem A, each $\varphi_{i}$ is a

convex combination of normalized functions of positive type $\psi_{i}^{(1)}, \psi_{i}^{(2)}$ with $\psi_{i}^{(1)}=1$ on $G(k)^{+}$and $\psi_{i}^{(2)}=0$ outside $Z(G)(k)$. Since $\tilde{\chi}=0$ outside $Z(G)(k)$, this implies that $\varphi_{i}=0$ outside $Z(G)(k)$ for $i=1,2$.

On the other hand, since $\left.\tilde{\chi}\right|_{Z(G)(k)}=\chi \in \operatorname{Char}(Z(G)(k))$, the functions $\varphi_{1}$ and $\varphi_{2}$ coincide with $\chi$ on $Z(G)(k)$. Hence $\varphi_{1}=\varphi_{2}=\tilde{\chi}$.

\section{EXtension OF THE RESUlts AND PROOFs OF COROLlaRies}

5.1. Extension to semi-simple groups. We extend Corollary B to semi-simple groups. First recall the following general result (see [Tho64, Satz]). Let $\Gamma=\Gamma_{1} \times \cdots \times \Gamma_{n}$ be a direct product of discrete groups. Then

$$
\operatorname{Char}(\Gamma)=\left\{\varphi_{1} \otimes \cdots \otimes \varphi_{n} \mid \varphi_{1} \in \operatorname{Char}\left(\Gamma_{1}\right), \ldots \varphi_{n} \in \operatorname{Char}\left(\Gamma_{n}\right)\right\},
$$

where $\left(\varphi_{1} \otimes \cdots \otimes \varphi_{n}\right)\left(\gamma_{1}, \ldots, \gamma_{2}\right)=\varphi_{1}\left(\gamma_{1}\right) \cdots \varphi_{n}\left(\gamma_{n}\right)$.

Let $G$ be a connected semi-simple algebraic group defined over a field $k$. As in the case of simple groups, define $G(k)^{+}$as the subgroup of $G(k)$ generated by the unipotent radicals of parabolic subgroups of $G$ defined over $k$.

Let $G_{1}, \ldots, G_{r}$ be the connected almost $k$-simple and $k$-isotropic normal $k$-subgroups of $G$. Then $G(k)^{+}$is the almost direct product of the $G_{i}(k)^{+}$'s, that is, the product map

$$
p: G_{1}^{+}(k) \times \cdots \times G_{r}(k)^{+} \rightarrow G(k)^{+}
$$

is a surjective homomorphism with finite kernel (see [BT73, 6.2]). In particular, we may identify $\operatorname{Char}\left(G(k)^{+}\right)$with a subset of characters of $G_{1}^{+}(k) \times \cdots \times G_{r}(k)^{+}$.

The following result is an immediate consequence of these remarks.

Proposition 5.1. With $k, G, G_{1}, \ldots, G_{r}$, and $p$ as above, $\operatorname{Char}\left(G(k)^{+}\right)$ coincides with the set functions $\varphi$ of the form $\varphi=\varphi_{1} \otimes \cdots \otimes \varphi_{r}$, with $\varphi_{i} \in \operatorname{Char}\left(G_{i}(k)^{+}\right)$for every $1 \leq i \leq r$ and such that $\varphi=1$ on $\operatorname{ker} p$.

5.2. Proof of Corollary B, Let $\varphi \in \operatorname{Char}\left(G(k)^{+}\right)$. Then $\widetilde{\varphi} \in P(G(k))$ and $\widetilde{\varphi}$ is $G(k)^{+}$-invariant. Hence, by Theorem $\mathrm{A}$, $\widetilde{\varphi}$ is a convex combination of two functions $\varphi_{1}$ and $\varphi_{2}$ from $P(G(k))$ with $\varphi_{1}=1$ on $G(k)^{+}$ and $\varphi_{2}=0$ outside $Z(G)(k)$. Since $\varphi$ is indecomposable, it follows that either $\varphi=\mathbf{1}_{G(k)^{+}}$or $\varphi=\tilde{\chi}$ for some $\chi$ in the dual of $Z(G) \cap G(k)^{+}$. Since, as observed in Remark 1.3, $Z(G) \cap G(k)^{+}=Z\left(G(k)^{+}\right)$, this completes the proof. 
5.3. Proof of Corollary C. (i) Observe first that $P G(k)^{+}$is a socalled ICC group, that is, every conjugacy class, except $\{e\}$, is infinite. Indeed, by Tits simplicity theorem, $P G(k)^{+}$has no proper subgroup of finite index. This implies that $L\left(P G(k)^{+}\right)$is a factor (see Lemma 5.3.4 in [MvN43]). Using Corollary B, the claim is proved as in [PT16, Theorem 3.5].

(ii) Observe that $P G(k)^{+}$is countable, since $k$ is assumed to be countable. As $P G(k)^{+}$is an ICC group, the claim follows from Corollary B and [PT16, Theorem 3.2] (or [DM14, Theorem 2.12]).

5.4. Proof of Corollary D. Item (i) is an immediate consequence of Corollary B and Remark 1.3, iii.

To show Item (ii), let $k$ be a global field and let $G(k) \curvearrowright(X, \mu)$ be a measure preserving ergodic action on a probability space $(X, \mu)$. Let $X^{\prime}$ be the set of points $x \in X$ with finite $G(k)^{+}$-orbit. By Tits' simplicity theorem, $X^{\prime}$ coincides with the $G(k)^{+}$-fixed points in $X$. Since $X^{\prime}$ is $G(k)$-invariant, we have either $\mu\left(X^{\prime}\right)=1$ or $\mu\left(X^{\prime}\right)=0$, by ergodicity of $G(k) \curvearrowright(X, \mu)$. In the first case, the action of $G(k)$ factorizes through $G(k) / G(k)^{+}=G(k)_{\mathrm{ab}}$. In the second case, the $G(k)$-orbit of $\mu$-almost every point in $X$ is infinite. Since $G(k)$ is a countable ICC-group, it follows from Item (i) and the proof of [PT16, Theorem 3.2] that $G(k) \curvearrowright(X, \mu)$ is essentially free.

\section{REFERENCES}

[AGV14] M. Abért, Y. Glasner, and B. Virág, Kesten's theorem for invariant random subgroups, Duke Math. J. 163 (2014), no. 3, 465-488.

$\left[\mathrm{ABB}^{+} 17\right]$ M. Abert, N. Bergeron, I. Biringer, T. Gelander, N. Nikolov, J. Raimbault, and I. Samet, On the growth of $L^{2}$-invariants for sequences of lattices in Lie groups, Ann. of Math. (2) 185 (2017), no. 3, 711-790.

[BHV08] B. Bekka, P. de la Harpe, and A. Valette, Kazhdan's property (T), New Mathematical Monographs, vol. 11, Cambridge University Press, Cambridge, 2008.

[Bek07] B. Bekka, Operator-algebraic superridigity for $\mathrm{SL}_{n}(\mathbb{Z}), n \geq 3$, Invent. Math. 169 (2007), no. 2, 401-425.

[Bor91] A. Borel, Linear algebraic groups, Second, Graduate Texts in Mathematics, vol. 126, Springer-Verlag, New York, 1991.

[BT65] A. Borel and J. Tits, Groupes réductifs, Inst. Hautes Études Sci. Publ. Math. 27 (1965), 55-150.

[BT73] _ Homomorphismes "abstraits" de groupes algébriques simples, Ann. of Math. (2) 97 (1973), 499-571.

[Bou68] N. Bourbaki, Groupes et algèbres de Lie. Chapitres IV, V, VI, Hermann, Paris, 1968.

[Bow15] L. Bowen, Invariant random subgroups of the free group, Groups Geom. Dyn. 9 (2015), no. 3, 891-916. 
[Dix77] J. Dixmier, $C^{*}$-algebras, North-Holland Publishing Co., Amsterdam-New York-Oxford, 1977.

[DM14] A. Dudko and K. Medynets, Finite factor representations of HigmanThompson groups, Groups Geom. Dyn. 8 (2014), no. 2, 375-389.

[Gel18] T. Gelander, A lecture on invariant random subgroups, New directions in locally compact groups, 2018, pp. 186-204.

[Gil09] P. Gille, Le problème de Kneser-Tits, Astérisque 326 (2009), Exp. No. 983, vii, 39-81 (2010). Séminaire Bourbaki. Vol. 2007/2008.

[Gla17] Y. Glasner, Invariant random subgroups of linear groups, Israel J. Math. 219 (2017), no. 1, 215-270.

[Gli61] J. Glimm, Type I C ${ }^{*}$-algebras, Ann. of Math. (2) 73 (1961), 572-612.

[HM79] R. Howe and C. Moore, Asymptotic properties of unitary representations, J. Functional Analysis 32 (1979), no. 1, 72-96.

[Kir65] A. A. Kirillov, Positive-definite functions on a group of matrices with elements from a discrete field, Soviet. Math. Dokl. 6 (1965), 707-709.

[LP11] M. Larsen and R. Pink, Finite subgroups of algebraic groups, J. Amer. Math. Soc. 24 (2011), no. 4, 1105-1158.

[MvN43] F. J. Murray and J. von Neumann, On rings of operators. IV, Ann. of Math. (2) 44 (1943), 716-808.

[Ovč71] S. V. Ovčinnikov, Positive definite functions on Chevalley groups, Funct. Anal. Appl. 5 (1971), no. 1, 79-80.

[Pet14] J. Peterson, Character rigidity for lattices in higher-rank groups (2014), available at www.math.vanderbilt.edu/ peters10/rigidity.pdf.

[PT16] J. Peterson and A. Thom, Character rigidity for special linear groups, J. Reine Angew. Math. 716 (2016), 207-228.

[PR94] V. Platonov and A. Rapinchuk, Algebraic groups and number theory, Pure and Applied Mathematics, vol. 139, Academic Press, Inc., Boston, MA, 1994.

[SvN50] I. Segal and J. von Neumann, A theorem on unitary representations of semisimple Lie groups, Ann. of Math. (2) $\mathbf{5 2}$ (1950), 509-517.

[Sku76] H-L Skudlarek, Die unzerlegbaren Charaktere einiger diskreter Gruppen, Math. Ann. 223 (1976), 213-231.

[SZ94] G. Stuck and R. Zimmer, Stabilizers for ergodic actions of higher rank semisimple groups, Ann. of Math. (2) 139 (1994), no. 3, 723-747.

[Tho64a] E. Thoma, Über positiv-definite Klassenfunktionen abzählbarer Gruppen, Math. Z. 84 (1964), 389-402.

[Tho64b] _ , Die unzerlegbaren, positiv-definiten Klassenfunktionen der abzählbar unendlichen, symmetrischen Gruppe, Math. Z. 85 (1964), 40-61.

[Tho68] _ Eine Charakterisierung diskreter Gruppen vom Typ I, Invent. Math. 6 (1968), 190-196.

[Tit64] J. Tits, Algebraic and abstract simple groups, Ann. of Math. (2) 80 (1964), 313-329.

[Tit78] _ Groupes de Whitehead de groupes algébriques simples sur un corps (d'après V. P. Platonov et al.), Séminaire Bourbaki, 29e année (1976/77), 1978, pp. Exp. No. 505, pp. 218-236.

[Ver11] A. M. Vershik, Nonfree actions of countable groups and their characters, J. Math. Sci. (New York) 174 (2011), 1-6. 
Bachir Bekka, Univ Rennes, CNRS, IRMAR-UMR 6625, Campus Beaulieu, F-35042 Rennes Cedex, France

E-mail address: bachir.bekka@univ-rennes1.fr 